\title{
Effects of ion channels on the spike timing in the retinal ganglion cells
}

\author{
Yoshimi Kamiyama*, Yuichiro Sakuragi \\ From Nineteenth Annual Computational Neuroscience Meeting: CNS*2010 \\ San Antonio, TX, USA. 24-30 July 2010
}

The ganglion cells of the vertebrate retina form the pathway by which the retina communicates with the visual cortex. The ganglion cells convert the graded potentials into a pattern of spikes whose characteristics is modulated by the synaptic and membrane currents. The ganglion cells respond with precise and reliable spikes to randomly flickering light [1]. This feature could not be reproduced by the previous models $[2,3]$, described with the deterministic differential equations similar to the Hodgkin-Huxley formulation. We proposed a stochastic model of spike generation in the ganglion cells, based on discrete stochastic ion channels represented by Markov processes [3]. We modeled eight types of ion channels, i.e., $\mathrm{Na}, \mathrm{Ca}(\mathrm{T}), \mathrm{Ca}(\mathrm{L}), \mathrm{Kv}, \mathrm{A}, \mathrm{K}$ (Ca), h and leakage channels. The proposed model showed precise and reliable spikes to randomly fluctuating current. This result suggested that the stochastic properties of ion channels are critical in determining the reliability of the spike timing in the retinal ganglion cells. However, the underlying mechanisms of the spike timing reliability has not yet been understood [3,4]. In this work, we systematically analyzed the feature of the spike timing reliability and the role of each channel stochasticity through computer simulation.

We measured the reliability of the spike timing for a wide range of fluctuating input patterns by varying the mean and standard deviation. We applied the event synchronization algorithm to simulated spikes [5]. The reliability was obtained from all two combinations in the spikes of 30 trials. The reliability of the spike patterns was strongly correlated with characteristics of the fluctuating input. This indicates the existence of optimal input range for the reliability. In order to clarify the role of ion channel stochasticity, we analyzed the contribution of each channel in the spike reliability, by changing the single channel conductance in simulation without changing the total electrical characteristics of the cell membrane. For a particular channel, the single channel conductance was increased by a factor of 20 , the channel density was decreased by a factor of $1 / 20$. The simulated results showed that the spike reproducibility is much influenced by the potassium channels, $\mathrm{Kv}$ and $\mathrm{A}$, not by the sodium channel, Na. These results suggest that the stochastic properties of $\mathrm{Kv}$ and A channels play a key role in determining the precise spike timing of retinal ganglion cells.

Published: 20 July 2010

\section{References}

1. Keat J, Reinagel P, Reid RC, Meister M: Predicting every spike: a model for the responses of visual neurons. Neuron 2001, 30:803-817.

2. Fohlmeister JF, Miller RF: Impulse encoding mechanisms of ganglion cells in the tiger salamander retina. J Neurophysiol 1997, 78:1935-1947.

3. Kamiyama Y, Ishihara A, Aoyama T, Usui S: Simulation analyses of retinal cell responses. Modeling in the neurosciences Taylor \& Francis, 2nd 2005.

4. van Rossum MC, O'Brien BJ, Smith RG: Effects of noise on the spike timing precision of retinal ganglion cells. J Neurophysiol 2003, 89:2406-2419.

5. Quian Quiroga R, Kreuz T, Grassberger P: Event synchronization: A simple and fast method to measure synchronicity and time delay patterns. Phys Rev E Stat Nonlin Soft Matter Phys 2002, 66:041904.

doi:10.1186/1471-2202-11-S1-P162

Cite this article as: Kamiyama and Sakuragi: Effects of ion channels on the spike timing in the retinal ganglion cells. BMC Neuroscience 2010 11(Suppl 1):P162.

\footnotetext{
* Correspondence: kamiyama@ist.aichi-pu.ac.jp

Information Science and Technology, Aichi Prefectural University, Nagakute, Aichi, 480-1198, Japan
} 